\title{
Hawking Radiation of Black Rings from Anomalies
}

\author{
Bin Chen 1 \\ Department of Physics, Peking University, Beijing 100871, P.R. China \\ Wei $\mathrm{H}^{2}$ \\ Institute of Theoretical Physics, Chinese Academy of Sciences,Beijing 100080 , P.R. China \\ and Graduate School of Chinese Academy of Sciences,Beijing 100049 , P.R. China
}

\begin{abstract}
We derive Hawking radiation of 5-dimensional black rings from gauge and gravitational anomalies using the method proposed by Robinson and Wilczek. We find as in the black hole case, the problem could reduce to a $(1+1)$ dimensional field theory and the anomalies result in correct Hawking temperature for neutral, dipole and charged black rings.
\end{abstract}

\footnotetext{
${ }^{1}$ bchen01@pku.edu.cn

${ }^{2}$ weihe@itp.ac.cn
} 


\section{Introduction}

Hawking radiation has been one of the central issues in black hole quantum mechanics since its discovery[1]. Recently Robinson and Wilczek proposed a new derivation of Hawking radiation of black hole via anomalies cancellation[2]. They treated the simplest Schwarzschild black hole and found that the cancellation of the gravitational anomalies result in correct Hawking temperature. Subsequent works extend this method to charged Reissner-Nordström black hole in [3] by including gauge anomalies, and to rotating Kerr black hole in [4]. The other related works treating various black holes could be found in [6].

In the recent study of higher dimensional gravity, people find it is possible to have another class of black objects in $D \geq 5$ dimesion: black rings. Unlike black holes, their horizon topology is not spherical. Especially in five dimension explicit solutions have been found for neutral, charged, and supersymmetric black rings with horizon topology $S^{1} \times S^{2}$. It's interesting to see if Robinson-Wilczek method applies to black rings, and we find it indeed works. This seems natural because in some limit black rings become Myers-Perry black holes, and their Hawking radiation is given in [5] by Robinson-Wilczek method. We find that for five dimensional black rings the scalar field theory reduces to a $(1+1)$ dimensional

free field theory near the horizon and the cancellation of gravitational and gauge anomalies give us the correct Hawking temperature.

We review Robinson-Wilczek method briefly in the next section and present our results in section three. In section four we discuss the relation between Robinson-Wilczek method and the tunneling picture[13] to get the Hawking temperature.

\section{Hawking radiation and anomalies}

In a black hole background we must be careful to define the quantum state of a field theory. If we choose Boulware state as the vacuum state, that is, define positive frequency using Schwarzschild time, then at the horizon the energy-momentum tensor of ground state is divergent due to ingoing modes. One way to avoid this problem is to choose Unruh vacuum by defining positive frequency using Kruskal coordinate, then these problematic ingoing modes in Boulware vacuum are removed because they are excited states now.

Robinson and Wilczek take another viewpoint [2, 3]: at classical level we just discard these ingoing modes near the horizon because they can't affect region outside the horizon. Then the quantum field theory near the horizon is chiral and suffers from the gravitational anomalies, and the gauge anomalies if gauge fields are presented. In order to cancel the anomalies to preserve the symmetries, additional fluxes/currents should be included. These 
fluxes/currens are quantum effects of the classically irrelevant ingoing modes. The condition of the anomalies cancellation and the regularity condition at the horizon determine Hawking flux of charge and energy-momentum.

For several kinds of black rings which will be studied in the next section, the near horizon field theory reduces to a $(1+1)$ dimensional free field theory with a background metric in the following form:

$$
d s^{2}=-f(r) d t^{2}+\frac{1}{f(r)} d r^{2}
$$

with $f\left(r_{H}\right)=0$ at the horizon $r_{H}$. The quantum field theory in this small region $r_{H} \leq$ $r \leq r_{H}+\epsilon$ could be treated as chiral theory, and suffers from anomalies. The gravitational anomalies in two dimension take a simple form

$$
\nabla_{\mu} T_{\chi \nu}^{\mu}=-\frac{1}{96 \pi \sqrt{-g}} \epsilon^{\beta \delta} \partial_{\delta} \partial_{\alpha} \Gamma_{\nu \beta}^{\alpha}=\frac{1}{\sqrt{-g}} \partial_{\mu} N_{\nu}^{\mu}=\mathcal{B}_{\nu}
$$

If there are background gauge fields, there exist gauge anomalies

$$
\nabla_{\mu} J_{\chi}^{\mu}= \pm \frac{e^{2}}{4 \pi \sqrt{-g}} \epsilon^{\mu \nu} \partial_{\mu} A_{\nu}
$$

As the symmetries of the underlying theory: general covariance and gauge symmetry must be preserved, the anomalies in the region $r_{H} \leq r \leq r_{H}+\epsilon$ must be cancelled by extra currents/fluxes.

First we analyze the gauge anomalies. Introduce a step functions $\Theta_{+}=\Theta\left(r-r_{H}-\epsilon\right)$ and $H=1-\Theta_{+}$we can write the current as 3

$$
J^{i}=J_{(H)}^{i} H+J_{(o)}^{i} \Theta_{+} .
$$

Because we consider the stationary configurations we have conserved current

$$
\partial_{r} J_{(o)}^{r}=0
$$

and the anomalous current

$$
\partial_{r} J_{(H)}^{r}=\frac{e^{2}}{4 \pi} \partial_{r} A_{t}
$$

They are solved by

$$
J_{(o)}^{y}=c_{o} \quad J_{(H)}^{y}=c_{H}+\frac{e^{2}}{4 \pi}\left(A_{t}(r)-A_{t}\left(r_{H}\right)\right)
$$

where $c_{o}, c_{H}$ are integration constants. The $c_{o}$ is the value of the flux at infinity and $c_{H}$ is the value of the current of the outgoing modes at the horizon. The gauge invariance of the full theory demands

$$
-\delta_{\lambda} W=\int d^{2} x \lambda\left[\left(J_{(o)}^{r}-J_{(H)}^{r}+\frac{e^{2}}{4 \pi} A_{t}\right) \delta\left(r-r_{H}-\epsilon\right)+\partial_{r}\left(\frac{e^{2}}{4 \pi} A_{t} H\right)\right]=0
$$

\footnotetext{
${ }^{3}$ We don't take into account the region inside the horizon because it's causally disconnected.
} 
where $\lambda$ is the gauge parameter. The last term in the integral is cancelled by the quantum effects of the classically irrelevant ingoing modes. In the limit $\epsilon \rightarrow 0$ we have

$$
c_{o}=c_{H}-\frac{e^{2}}{4 \pi} A_{t}\left(r_{H}\right)
$$

In order to fix the constants we impose a boundary condition: the covariant current $\tilde{J}^{i}=$ $J^{i}+\frac{e^{2}}{4 \pi} A_{t}(r) H$ vanish at the horizon. This is the regularity condition as pointed out in the first reference of [4]. Then the integration constants are fixed

$$
c_{o}=2 c_{H}=-\frac{e^{2}}{2 \pi} A_{t}\left(r_{H}\right) .
$$

It represents Hawking flux of charge.

For the gravitational anomalies, we can write $T_{\nu}^{\mu}$ as

$$
T_{\nu}^{\mu}=T_{(H) \nu}^{\mu} H+T_{(o) \nu}^{\mu} \Theta_{+}
$$

For the metric (1) we have :

$$
\begin{aligned}
& N_{t}^{t}=N_{r}^{r}=0 \\
& N_{t}^{r}=\frac{1}{192 \pi}\left(f f^{\prime \prime}+f^{\prime 2}\right) \\
& N_{r}^{t}=\frac{1}{192 \pi} \frac{f^{\prime \prime} f-f^{\prime 2}}{f^{2}}
\end{aligned}
$$

where prime denotes derivative with respect to $r$.

Under diffeomorphism transformation $x \rightarrow x^{\prime}=x-\xi$, taking gauge fields into account we have

$$
-\delta_{\xi} W=\int d^{2} x \sqrt{-g_{(2)}} \xi^{\nu} \nabla_{\mu} T_{\nu}^{\mu}
$$

where

$$
\nabla_{\mu} T_{\nu}^{\mu}=F_{\mu \nu} J^{\mu}+A_{\nu} \nabla_{\mu} J^{\mu}+\mathcal{B}_{\nu}
$$

For the energy-momentum tensor in radial direction, in the region $r>r_{H}+\epsilon$ we have

$$
\partial_{r} T_{(o) t}^{r}=F_{r t} J_{(o)}^{r}
$$

in the region $r_{H} \leq r \leq r_{H}+\epsilon$, since $\nabla_{\mu} J^{\mu} \neq 0, N_{t}^{r} \neq 0$, we have

$$
\partial_{r} T_{(H) t}^{r}=F_{r t} J_{(H)}^{r}+A_{t} \partial_{r} J_{(H)}^{r}+\partial_{r} N_{t}^{r}
$$

They are solved by

$$
\begin{aligned}
T_{(o) t}^{r} & =a_{o}+c_{o} A_{t}(r) \\
T_{(H) t}^{r} & =a_{H}+\int_{r_{H}}^{r} \partial_{r}\left(c_{o} A_{t}+\frac{e^{2}}{4 \pi} A_{t}^{2}+N_{t}^{r}\right) d r
\end{aligned}
$$


The general covariance demands

$$
\int d^{2} x \xi^{t}\left[\partial_{r}\left(A_{t} J_{(H)}^{r} H\right)+\partial_{r}\left(N_{t}^{r} H\right)+\left(T_{(o) t}^{r}-T_{(H) t}^{r}-A_{t} J_{(H)}^{r}+N_{t}^{r}\right) \delta\left(r-r_{H}-\epsilon\right)\right]=0
$$

The first term in the integral should be cancelled by the quantum effect of the ingoing modes. Taking $\epsilon \rightarrow 0$ limit, we get

$$
a_{o}=a_{H}+\frac{e^{2}}{4 \pi} A_{t}^{2}\left(r_{H}\right)-N_{t}^{r}\left(r_{H}\right)
$$

Imposing a vanishing condition for the covariant energy-momentum $\tilde{T}_{t}^{r}=T_{t}^{r}+\left(f f^{\prime \prime}-\right.$ $\left.2 f^{\prime 2}\right) / 192 \pi$ on the horizon, we get

$$
a_{H}=2 N_{t}^{r}\left(r_{H}\right) \quad a_{o}=\frac{e^{2}}{4 \pi} A_{t}^{2}\left(r_{H}\right)+N_{t}^{r}\left(r_{H}\right)
$$

The $a_{o}$ represents Hawking flux of energy-momentum.

The flux $\Phi=N_{t}^{r}\left(r_{H}\right)$ represents a thermal flux due to Hawking radiation, it's related to temperature as $\Phi=\frac{\pi}{12} T^{2}$. Therefore we can get Hawking temperature

$$
T=\frac{f^{\prime}\left(r_{H}\right)}{4 \pi}
$$

\section{Hawking radiation of 5-dimensional black rings from anomalies}

\subsection{Neutral black ring}

The five dimensional neutral black ring was first found in [7] as a vacuum solution of five dimensional general relativity. Black rings obey similar thermodynamics laws as black holes, while lose uniqueness because in some range of their parameter space there are more than one solution with the same conserved charges. For more aspects about five dimensional black rings, see the review [12].

The metric of the neutral black ring is (we use the coordinates given in [10])

$$
\begin{aligned}
d s^{2}= & -\frac{F(y)}{F(x)}\left(d t-C R \frac{1+y}{F(y)} d \psi\right)^{2} \\
& +\frac{R^{2}}{(x-y)^{2}} F(x)\left[-\frac{G(y)}{F(y)} d \psi^{2}-\frac{d y^{2}}{G(y)}+\frac{d x^{2}}{G(x)}+\frac{G(x)}{F(x)} d \phi^{2}\right]
\end{aligned}
$$

with functions

$$
F(\xi)=1+\lambda \xi \quad G(\xi)=\left(1-\xi^{2}\right)(1+\nu \xi)
$$


and constant

$$
C=\sqrt{\lambda(\lambda-\nu) \frac{1+\lambda}{1-\lambda}}, \quad 0<\nu \leq \lambda<1
$$

the coordinates $\psi, \phi$ are two cycles of the black ring and $x, y$ take values

$$
-1 \leq x \leq 1, \quad-\infty \leq y \leq-1
$$

The center of the black ring is located at $y=-\infty$, and $x \rightarrow-1, y \rightarrow-1$ approaches the asymptotic infinity. The horizon is at $y=y_{H}=-\frac{1}{\nu}$ with the topology $S^{1} \times S^{2}$. The mass of the black ring is

$$
M=\frac{3 \pi R^{2}}{4 G} \frac{\lambda}{1-\nu}
$$

In order to prevent the contraction the ring must rotate along the $\psi$ direction, the angular momentum is

$$
J=\frac{\pi R^{3}}{2 G} \frac{\sqrt{\lambda(\lambda-\nu)(1+\lambda)}}{(1-\nu)^{2}}
$$

The neutral black rings are parameterized by their mass and angular momentum, or by $\lambda$ and $\nu$.

Now let us show that near the horizon a scalar field theory reduces to a $(1+1)$ dimensional free field theory. For black ring metric we have

$$
\sqrt{-g}=\frac{R^{4} F(x)}{(x-y)^{4}}
$$

and the non-zero components of the inverse metric are

$$
\begin{aligned}
g^{t t} & =-\frac{C^{2}(x-y)^{2}(1+y)^{2}}{F(x) F(y) G(y)}-\frac{F(x)}{F(y)} \quad g^{t \psi}=-\frac{C(x-y)^{2}(1+y)}{R F(x) G(y)} \\
g^{\psi \psi} & =-\frac{(x-y)^{2} F(y)}{R^{2} F(x) G(y)} \quad g^{x x}=\frac{(x-y)^{2} G(x)}{R^{2} F(x)} \\
g^{y y} & =-\frac{(x-y)^{2} G(y)}{R^{2} F(x)} \quad g^{\phi \phi}=\frac{(x-y)^{2}}{R^{2} G(x)}
\end{aligned}
$$

For a scalar field $\varphi$ in the black ring background, the action is

$$
\begin{aligned}
S[\varphi]= & \int d^{5} x\left(\varphi \partial_{i} \sqrt{-g} g^{i j} \partial_{j} \varphi+\sqrt{-g} V_{\text {int }}(\varphi)\right) \\
= & \int d^{5} x \varphi\left[-\left(\frac{C^{2} R^{4}(1+y)^{2}}{(x-y)^{2} F(y) G(y)}+\frac{R^{4} F(x)^{2}}{(x-y)^{4} F(y)}\right) \partial_{t}^{2}-2 \frac{C R^{3}(1+y)}{(x-y)^{2} G(y)} \partial_{t} \partial_{\psi}\right. \\
& \left.-\frac{R^{2} F(y)}{(x-y)^{2} G(y)} \partial_{\psi}^{2}+\partial_{x} \frac{R^{2} G(x)}{(x-y)^{2}} \partial_{x}-\partial_{y} \frac{R^{2} G(y)}{(x-y)^{2}} \partial_{y}+\frac{R^{2} F(x)}{(x-y)^{2} G(x)} \partial_{\phi}^{2}\right] \varphi \\
& +\int d^{5} x \frac{R^{4} F(x)}{(x-y)^{4}} V_{\text {int }}(\varphi)
\end{aligned}
$$


We expand the scalar field as

$$
\varphi=\sum_{k, l} \frac{1}{2 \pi} \varphi^{(k l)}(t, x, y) e^{i k \phi} e^{i l \psi}
$$

where $k, l$ are integers because $\phi, \psi$ are periodic coordinates. Put it into the action and take the near horizon limit $y \rightarrow y_{H}, G(y) \rightarrow 0$, leaving the dominant terms in the action we get

$$
S[\varphi]=\int d^{5} x \varphi^{(k l)} \frac{R^{2}}{(x-y)^{2}}\left[-\frac{C^{2} R^{2}(1+y)^{2}}{F(y) G(y)}\left(\partial_{t}+i \frac{l F(y)}{C R(1+y)}\right)^{2}-\partial_{y} G(y) \partial_{y}\right] \varphi^{(k l)}
$$

As in black hole cases, the potential term for the scalar field is suppressed. Moreover, the terms involving $\partial_{x}$ are also suppressed and do not appear in the action. Therefore we can further expand function $\varphi^{(k l)}(t, x, y)$ in terms of $x$. As $x=\cos \theta$ in polar coordinate, an appropriate expansion function is the Legendre polynomial $P_{n}(x)$,

$$
\varphi^{(k l)}(t, x, y)=\sum_{n} \varphi^{(k l n)}(t, y) P_{n}(x)
$$

Moving $P_{n}(x)$ to the left of the operator, we integrate over $x$ and have :

$$
\int_{-1}^{1} d x \frac{P_{m}(x) P_{n}(x)}{(x-y)^{2}}=a_{m n}(y)
$$

Then the action can be written as

$$
\begin{aligned}
S[\varphi]= & \int d t d y \sum_{k, l, m, n} a_{m n}(y) \frac{C R^{3}(1+y)}{\sqrt{-F(y)}} \times \varphi^{(k l m)}(t, y) \\
& {\left[\frac{C R(1+y)}{\sqrt{-F(y)} G(y)}\left(\partial_{t}+i l \frac{F(y)}{C R(1+y)}\right)^{2}-\partial_{y} \frac{\sqrt{-F(y)}}{C R(1+y)} G(y) \partial_{y}\right] \varphi^{(k l n)}(t, y) }
\end{aligned}
$$

In the above action we can treat $\frac{F(y)}{C R(1+y)}$ as an effective gauge field $A_{t}(y)$ and $l$ serves as the gauge coupling constant. The coefficient $a_{m n}(y)$ is symmetric about $m, n$. We can further absorb the factor $a_{m n}(y) \frac{C R^{3}(1+y)}{\sqrt{-F(y)}}$ in to $\varphi^{(k l n)}(t, y)$ and define a new field $\tilde{\varphi}^{(k l n)}(t, y)$, then the action can be written in a canonical form

$$
\begin{aligned}
S[\varphi]= & \int d t d y \sum_{k, l, n} \tilde{\varphi}^{(k l n)}(t, y) \\
& \times\left[\frac{C R(1+y)}{\sqrt{-F(y)} G(y)}\left(\partial_{t}+i l \frac{F(y)}{C R(1+y)}\right)^{2}-\partial_{y} \frac{\sqrt{-F(y)}}{C R(1+y)} G(y) \partial_{y}\right] \tilde{\varphi}^{(k l n)}(t, y)
\end{aligned}
$$

It's clear we get an infinite set of effective free massless fields $\tilde{\varphi}^{(k l m)}(t, y)$ in $(1+1)$ dimension with metric

$$
d s^{2}=-f(y) d t^{2}+\frac{1}{f(y)} d y^{2}
$$


where

$$
f(y)=\frac{\sqrt{-F(y)}}{C R(1+y)} G(y)
$$

together with a background $U(1)$ gauge field

$$
A_{t}(y)=-\frac{F(y)}{C R(1+y)}
$$

In the near horizon region $y=-\frac{1}{\nu}+\epsilon$ we have $F(y)<0, G(y)<0,(1+y)<0$, so $t$ is timelike and $y$ is spacelike.

It is remarkable that the essential point in the above discussion is the absence of the derivatives with respect to $x$ in the reduced action (32). This indicates that there is no dynamics in $x$ direction. Effectively we may take $x$ as just a parameter to label the field. This is the reason why we get a two-dimensional free scalar field theory in the near horizon limit.

The analysis of the gauge and gravitational anomalies in this two dimensional theory is the same as in section 2. For the gauge anomalies we have

$$
J_{(o)}^{y}=c_{o} \quad J_{(H)}^{y}=c_{H}+\frac{l^{2}}{4 \pi}\left(A_{t}(y)-A_{t}\left(y_{H}\right)\right)
$$

with

$$
c_{o}=2 c_{H}=-\frac{l^{2}}{2 \pi} A_{t}\left(y_{H}\right)=\frac{l^{2}}{2 \pi} \Omega_{H} .
$$

We have written $-A_{t}\left(y_{H}\right)$ as $\Omega_{H}$ where $\Omega_{H}$ is the angular velocity at the horizon.

For the gravitational anomalies we have

$$
\begin{aligned}
T_{(o) t}^{y} & =a_{o}+c_{o} A_{t}(y) \\
T_{(H) t}^{y} & =a_{H}+\left.\left(c_{o} A_{t}+\frac{l^{2}}{4 \pi} A_{t}^{2}+N_{t}^{y}\right)\right|_{y_{H}} ^{y}
\end{aligned}
$$

with

$$
a_{o}=\frac{l^{2}}{4 \pi} \Omega_{H}^{2}+N_{t}^{y}\left(y_{H}\right) \quad a_{H}=2 N_{t}^{y}\left(y_{H}\right)
$$

From the relation $\Phi=\frac{\pi}{12} T^{2}$, where $\Phi$ is the flux $N_{t}^{y}\left(y_{H}\right)$, we get the Hawking temperature of the black ring

$$
T=\frac{f^{\prime}\left(y_{H}\right)}{4 \pi}=\frac{1}{4 \pi R} \frac{1+\nu}{\sqrt{\lambda \nu}} \sqrt{\frac{1-\lambda}{1+\lambda}} .
$$

\subsection{Dipole black ring}

The five dimensional dipole black rings was first constructed in [10], its metric is of the form

$$
\begin{aligned}
d s^{2} & =-\frac{F(y) H(x)}{F(x) H(y)}\left(d t-C R \frac{1+y}{F(y)} d \psi\right)^{2} \\
& +\frac{R^{2} F(x) H(x) H(y)^{2}}{(x-y)^{2}}\left[-\frac{G(y)}{F(y) H(y)^{3}} d \psi^{2}-\frac{d y^{2}}{G(y)}+\frac{d x^{2}}{G(x)}+\frac{G(x)}{F(x) H(x)^{3}} d \phi^{2}\right]
\end{aligned}
$$


with $F(\xi), G(\xi)$ being defined as before, and the new function $H(\xi)$ being:

$$
H(\xi)=\left[H_{1}(\xi) H_{2}(\xi) H_{3}(\xi)\right]^{\frac{1}{3}} \quad \text { with } \quad H_{i}(\xi)=1-\mu_{i} \xi
$$

where $0 \leq \mu_{i}<1, i=1,2,3$. The $\mu_{i}$ 's are parameters related to the dipoles of black ring. The scalar and gauge fields are

$$
X^{i}=\frac{H(x) H_{i}(y)}{H(y) H_{i}(x)} \quad A_{i}=C_{i} R \frac{1+x}{H_{i}(x)} d \phi
$$

where $C_{i}$ 's are of the same form as $C$ but with $\lambda \rightarrow-\mu_{i}$. As the gauge fields are magnetic, they represent circularly distributed monopole charges. This kind of black ring has no conserved gauge charges while allows continuous value of dipoles:

$$
q_{i}=\frac{1}{2 \pi} \int_{S^{2}} d A^{i}
$$

so dipole black rings continuously violate the uniqueness. These dipole charges are not conserved charges of black rings, but they do appear in the first law of thermodynamics of black rings[10, 11].

We can analyze the scalar action in this dipole black ring background and expand $\varphi$ in the same way as in the neutral case, after field redefinition we can get a two dimensional massless free scalar field theory with action similar with (36). We will not list the details but just give the final form:

$$
\begin{aligned}
S[\varphi]= & \int d t d y \sum_{k, l, n} \tilde{\varphi}^{(k l n)}(t, y)\left[\frac{C R(1+y) H(y)^{3 / 2}}{\sqrt{-F(y)} G(y)}\right. \\
& \left.\left(\partial_{t}+\frac{i l F(y)}{C R(1+y)}\right)^{2}-\partial_{y} \frac{\sqrt{-F(y)} G(y)}{C R(1+y) H(y)^{3 / 2}} \partial_{y}\right] \tilde{\varphi}^{(k l n)}(t, y) .
\end{aligned}
$$

Note that gauge fields $A_{i \phi}$ do not contribute dominant terms in the action. The action is similar to the neutral case except a new $H(y)^{3 / 2}$ factor.

The back ground metric is of the same form as (37) with

$$
f(y)=\frac{\sqrt{-F(y)}}{C R(1+y) H(y)^{3 / 2}} G(y)
$$

and together with a gauge field

$$
A_{t}(y)=-\frac{F(y)}{C R(1+y)}
$$

Note that $A_{i \phi}$ 's are not contained in our $(1+1)$ dimensional theory because they are magnetic, and their electric dual are two-form fields that do not couple to point particles. In fact, $A_{i \phi}$ 's behave as three scalar fields in the near horizon 2-dimensional background. 
The analysis of both the gauge and the gravitational anomalies is parallel with the neutral case except their $f(y)$ 's are different. The Hawking temperature of the dipole black ring is

$$
T=\frac{f^{\prime}\left(y_{H}\right)}{4 \pi}=\frac{1}{4 \pi R} \frac{1+\nu}{\sqrt{\lambda \nu}} \sqrt{\frac{1-\lambda}{1+\lambda}} \frac{1}{\sqrt{\prod_{i}\left(1+\frac{\mu_{i}}{\nu}\right)}}
$$

\subsection{Charged black ring}

The rotating black ring with a single electric charge was first constructed in [8] as a solution of low energy effective action of heterotic string, black rings with two and three charges were given in [9]. We only treat the single-charged black ring in this subsection. For black rings with two or three charges, the discussion are similar because charged black rings have very similar structure.

The metric of the single-charged ring can be written as 4

$$
\begin{aligned}
d s^{2}= & -\frac{F(y)}{F(x) K(x, y)^{2}}\left(d t-C R \frac{1+y}{F(y)} \cosh ^{2} \alpha d \psi\right)^{2} \\
& +\frac{R^{2}}{(x-y)^{2}} F(x)\left[-\frac{G(y)}{F(y)} d \psi^{2}-\frac{d y^{2}}{G(y)}+\frac{d x^{2}}{G(x)}+\frac{G(x)}{F(x)} d \phi^{2}\right]
\end{aligned}
$$

where functions $F(\xi), G(\xi)$ are defined as before, and $K(x, y)$ is defined as

$$
K(x, y)=1+\frac{\lambda(x-y)}{F(x)} \sinh ^{2} \alpha
$$

where $\alpha$ is the parameter represents the electric charge.

The dilation field is

$$
e^{-\Phi}=K(x, y)
$$

and the gauge fields are

$$
A_{t}=\frac{\lambda(x-y) \sinh \alpha \cosh \alpha}{F(x) K(x, y)}, \quad A_{\psi}=\frac{C R(1+y) \sinh \alpha \cosh \alpha}{F(x) K(x, y)}
$$

with electric charge

$$
Q=\frac{2 \sinh 2 \alpha}{3\left(1+\frac{4}{3} \sinh ^{2} \alpha\right)} M
$$

there is also a 2 -form $B_{t \psi}$ field which indicates that the black ring carries local fundamental string charge, but it is irrelevant to our discussion because it does not couple to point particles.

\footnotetext{
${ }^{4}$ Metric in this form can be obtained from D1-D5 solution in 6-dimension given in apendix.A of [9] by a dimensional reduction in $z$-direction and set charges equal $\alpha_{1}=\alpha_{5}=\alpha$. In order to write it in the form consistent with neutral and dipole cases a further coordinates transformation is needed [12]: $x \rightarrow \frac{x+\lambda}{1+\lambda x}, y \rightarrow$ $\frac{y+\lambda}{1+\lambda y},(\psi, \phi)=\frac{1-\lambda \nu}{\sqrt{1-\lambda^{2}}}(\psi, \phi), \lambda \rightarrow \lambda, \nu \rightarrow \frac{\lambda-\nu}{1-\lambda \nu}$.
} 
The action of a scalar field in this black ring background is

$$
S[\varphi]=\int d^{5} x\left(\varphi \mathcal{D}_{i} \sqrt{-g} g^{i j} \mathcal{D}_{j} \varphi+\sqrt{-g} V_{i n t}(\varphi)\right) .
$$

The terms containing covariant derivatives appear as

$$
\frac{R^{2}}{(x-y)^{2} K(x, y)}\left[-\frac{C^{2} R^{2}(1+y)^{2} \cosh ^{4} \alpha}{F(y) G(y)} \mathcal{D}_{t}^{2}-2 \frac{C R(1+y) \cosh ^{2} \alpha}{G(y)} \mathcal{D}_{t} \mathcal{D}_{\psi}-\frac{F(y)}{G(y)} \mathcal{D}_{\psi}^{2}\right]
$$

We expand $\varphi$ as (31) and put it into the action, after taking near horizon limit $y \sim y_{H}+\epsilon$ we get the dominant action for field $\varphi^{(k l)}(t, x, y)$ :

$$
\begin{aligned}
S[\varphi]= & \int d^{5} x \varphi^{(k l)} \frac{R^{2}}{(x-y)^{2} K(x, y)}\left[-\frac{C^{2} R^{2}(1+y)^{2} \cosh ^{4} \alpha}{F(y) G(y)}\left(\partial_{t}+i e A_{t}\right.\right. \\
& \left.\left.+\frac{i e A_{\psi} F(y)}{C R(1+y) \cosh ^{2} \alpha}+\frac{i l F(y)}{C R(1+y) \cosh ^{2} \alpha}\right)^{2}-\partial_{y} G(y) \partial_{y}+\partial_{x} G(x) \partial_{x}\right] \varphi^{(k l)} \\
= & \int d^{5} x \varphi^{(k l)} \frac{R^{2}}{(x-y)^{2} K(x, y)}\left[-\frac{C^{2} R^{2}(1+y)^{2} \cosh ^{4} \alpha}{F(y) G(y)}\right. \\
& \left.\left(\partial_{t}+i e \tanh \alpha+\frac{i l F(y)}{C R(1+y) \cosh ^{2} \alpha}\right)^{2}-\partial_{y} G(y) \partial_{y}\right] \varphi^{(k l)} .
\end{aligned}
$$

In the second step we discard $\partial_{x}$ part because the $x$-dependence coming from $A_{t}(x, y)$ and $A_{\psi}(x, y)$ cancels exactly.

Now as the operator in the bracket does not depend on $x$ anymore, we can expand $\varphi^{(k l)}(t, x, y)$ as before $\varphi^{(k l)}(t, x, y)=\sum_{n} \varphi^{(k l n)}(t, y) P_{n}(x)$. After integrating $x$

$$
\int_{-1}^{1} d x \frac{P_{m}(x) P_{n}(x)}{(x-y)^{2} K(x, y)}=b_{m n}(y)
$$

the action becomes

$$
\begin{aligned}
S[\varphi]= & \int d t d y \frac{C R^{3}(1-y) \cosh ^{2} \alpha}{\sqrt{-F(y)}} b_{m n}(y) \varphi^{(k l m)}(t, y)\left[\frac{C R(1+y) \cosh ^{2} \alpha}{\sqrt{-F(y)} G(y)}\right. \\
& \left.\left(\partial_{t}+i e \tanh \alpha+i l \frac{F(y)}{C R(1+y) \cosh ^{2} \alpha}\right)^{2}-\partial_{y} \frac{\sqrt{-F(y)} G(y)}{C R(1+y) \cosh ^{2} \alpha} \partial_{y}\right] \varphi^{(k l n)}(t, y) .
\end{aligned}
$$

After field redefinition, we can write the action in the canonical form. The action describe an infinite collection of massless scalar fields in a $(1+1)$ dimensional background, whose metric is of the form (37) with

$$
f(y)=\frac{\sqrt{-F(y)}}{C R(1+y) \cosh ^{2} \alpha} G(y)
$$

and coupling to two $U(1)$ fields

$$
A_{t}^{(1)}=-\tanh \alpha, \quad A_{t}^{(2)}(y)=-\frac{F(y)}{C R(1+y) \cosh ^{2} \alpha} .
$$


Note that $A_{t}^{(2)}\left(y_{H}\right)=-\Omega_{H}$ where $\Omega_{H}$ is the angular velocity at the horizon.

Extend the gauge anomalies analysis to more than one gauge fields is straightforward. The anomaly equations for electric currents are

$$
\begin{aligned}
\partial_{y} J_{(H)}^{(1) y} & =\frac{e}{4 \pi} \partial_{y}\left(e A_{t}^{(1)}+l A_{t}^{(2)}\right) \\
\partial_{y} J_{(H)}^{(2) y} & =\frac{l}{4 \pi} \partial_{y}\left(e A_{t}^{(1)}+l A_{t}^{(2)}\right)
\end{aligned}
$$

As $A_{t}^{(1)}$ is a constant it in fact does not contribute anomalies in the region $y_{H} \leq y \leq y_{H}+\epsilon$, so we have

$$
J_{(o)}^{(1) y}=c_{o}^{(1)} \quad J_{(H)}^{(1) y}=c_{H}^{(1)}+\frac{e l}{4 \pi}\left(A_{t}^{(2)}(y)-A_{t}^{(2)}\left(y_{H}\right)\right) .
$$

The gauge invariance of $A_{t}^{(y)}$ demands

$$
c_{o}^{(1)}=c_{H}^{(1)}-\frac{e}{4 \pi}\left(e A_{t}^{(1)}\left(y_{H}\right)+l A_{t}^{(2)}\left(y_{H}\right)\right)
$$

and the regular boundary condition leads to

$$
c_{o}^{(1)}=\frac{e}{2 \pi}\left(e \tanh \alpha+l \Omega_{H}\right)
$$

Similarly we can get $J^{(2) y}$.

For gravitational anomalies we have

$$
\begin{aligned}
T_{(o) t}^{y} & =a_{o}+c_{o}^{(1)} A_{t}^{(1)}(y)+c_{o}^{(2)} A_{t}^{(2)}(y) \\
T_{(H) t}^{y} & =a_{H}+\int_{y_{H}}^{y} \partial_{y}\left(c_{o}^{(2)} A_{t}^{(2)}+\frac{l^{2}}{4 \pi} A_{t}^{(2) 2}+N_{t}^{y}\right) d y .
\end{aligned}
$$

The general covariance demands

$$
a_{o}=a_{H}-c_{H}^{(1)} A_{t}^{(1)}\left(y_{H}\right)-c_{H}^{(2)} A_{t}^{(2)}\left(y_{H}\right)-N_{t}^{y}\left(y_{H}\right)
$$

A covariance boundary condition fixes

$$
a_{H}=2 N_{t}^{y}\left(y_{H}\right), \quad a_{o}=\frac{1}{4 \pi}\left(e \tanh \alpha+l \Omega_{H}\right)^{2}+N_{t}^{y}\left(y_{H}\right)
$$

The Hawking temperature is given by

$$
T=\frac{f^{\prime}\left(y_{H}\right)}{4 \pi}=\frac{1}{4 \pi R \cosh ^{2} \alpha} \frac{1+\nu}{\sqrt{\lambda \nu}} \sqrt{\frac{1-\lambda}{1+\lambda}} .
$$

In the extremal limit $\alpha \rightarrow \infty$, we have $Q \rightarrow M, T \rightarrow 0$. 
Now let's compare our results with the black body radiation of black rings at temperature $T=\frac{1}{\beta}$, in order to avoid superradiance problem we only consider fermions. The Plank distribution of fermions with energy $\omega$, charge $e$ and angular momentum $l$ is

$$
N_{e, l}(\omega)=\frac{1}{e^{\beta\left(\omega-e \Phi-l \Omega_{H}\right)}+1}
$$

where $\Phi$ is the co-rotating electric chemical potential at the horizon and $\Omega_{H}$ is the angular velocity at the horizon. The Killing vector of black ring background is $\xi=\partial_{t}+\Omega_{H} \partial_{\psi}$, so we have

$$
\Phi=\xi^{\mu} A_{\mu}=A_{t}\left(x, y_{H}\right)+\Omega_{H} A_{\psi}\left(x, y_{H}\right)=\tanh \alpha .
$$

Then the Hawking flux of electric charge, angular momentum and energy-momentum tensor are

$$
\begin{aligned}
J_{e} & =e \int_{0}^{\infty} \frac{d \omega}{2 \pi}\left(N_{e, l}(\omega)-N_{-e,-l}(\omega)\right)=\frac{e}{2 \pi}\left(e \tanh \alpha+l \Omega_{H}\right) \\
J_{l} & =l \int_{0}^{\infty} \frac{d \omega}{2 \pi}\left(N_{e, l}(\omega)-N_{-e,-l}(\omega)\right)=\frac{l}{2 \pi}\left(e \tanh \alpha+l \Omega_{H}\right) \\
J_{E} & =\int_{0}^{\infty} \frac{d \omega}{2 \pi} \omega\left(N_{e, l}(\omega)+N_{-e,-l}(\omega)\right)=\frac{1}{4 \pi}\left(e \tanh \alpha+l \Omega_{H}\right)^{2}+\frac{\pi}{12 \beta^{2}}
\end{aligned}
$$

They agree with the results (68,71) derived from anomalies cancellation. Set $e=0$ we get the results for neutral black ring.

\section{Relation to tunneling picture}

In [13] Parrikh and Wilczek showed how to derive Hawking temperature in the tunneling picture. We need to calculate the tunneling probability of a particle tunnels from $r_{i n}=$ $r_{H}(M)-\epsilon$ inside the initial horizon to $r_{\text {out }}=r_{H}(M-\omega)+\epsilon$ outside the final horizon in the Painleve coordinate. Under WKB approximation, it is:

$$
\Gamma \sim e^{-2 \operatorname{Im} S}
$$

with

$$
\operatorname{Im} S=\operatorname{Im} \int_{r_{\text {in }}}^{r_{\text {out }}} p_{r} d r=\operatorname{Im} \int_{0}^{\omega} \int_{r_{\text {in }}}^{r_{\text {out }}} \frac{d r}{\dot{r}} d(-\omega) .
$$

Here $\omega$ is the energy of the radiated particle, $M(M-\omega)$ is the mass of black hole before (after) emission. And $\dot{r}$ is the radial null geodesic, it's a function of $r$ and conserved charges such as $M, Q, J$. As $\omega \ll M$, to first order the above expression is approximately:

$$
\operatorname{Im} S \simeq \omega \operatorname{Im} \int_{r_{\text {out }}}^{r_{\text {in }}} \frac{d r}{\dot{r}}
$$


Let us apply the above analysis to the black rings studied above. For simplicity, we focus on the neutral black ring and consider the effective two-dimensional near-horizon metric (37) obtained in Robinson-Wilczek method. Transforming $t$ coordinate as

$$
t \rightarrow t-\int \frac{C R(1+y)}{\sqrt{-F(y)} G(y)} \sqrt{1-G(y)} d y
$$

we can rewrite the metric in the Painleve form

$$
d s^{2}=-\frac{\sqrt{-F(y)} G(y)}{C R(1+y)} d t^{2}+2 \sqrt{1-G(y)} d t d y+\frac{C R(1+y)}{\sqrt{-F(y)}} d y^{2}
$$

Consider a uncharged particle to avoid the influence of electromagnetic fields, the null geodesics is

$$
\dot{y}=\frac{\sqrt{-F(y)}}{C R(1+y)}( \pm 1-\sqrt{1-G(y)})
$$

where \pm correspond to ougoing/ingoing geodesics.

Performing the integral (78) with outgoing geodesic, we have

$$
\begin{aligned}
\operatorname{Im} S & \simeq \omega \frac{C R\left(1-\frac{1}{\nu}\right)}{\sqrt{-F\left(-\frac{1}{\nu}\right)}} \times 2 \pi \operatorname{Res}_{y_{H}} \frac{1}{1-\sqrt{1-G(y)}} \\
& \simeq \omega \frac{C R\left(1-\frac{1}{\nu}\right)}{\sqrt{-F\left(-\frac{1}{\nu}\right)}} \times 2 \pi \frac{2 \nu}{\nu^{2}-1}
\end{aligned}
$$

From $\Gamma \sim e^{-2 \operatorname{ImS}} \sim e^{-\frac{\omega}{T}}$, we get the Hawking temperature

$$
T=\frac{1}{4 \pi R} \frac{1+\nu}{\sqrt{\lambda \nu}} \sqrt{\frac{1-\lambda}{1+\lambda}} .
$$

The situations for dipole and charged black rings are similar.

\section{Conclusion}

In this paper, we extended Robinson-Wilczek method to the five dimensional black rings and got their Hawking temperature and fluxes correctly. As in the rotating black hole case, near the horizon the field theory reduces to a $(1+1)$ dimensional free field theory coupled to gauge fields after appropriate field redefinition. From the cancellation of the gauge and gravitational anomalies, we obtain the correct Hawking temperature and radiation. The same two dimensional metric also determines the null geodesics in the tunneling method and leads to the same Hawking temperature. This reflects the fact that the Hawking radiation is determined universally by the horizon properties. 
From studying various black holes and black rings here, we expect for stationary black objects in various dimensions with more complicated horizon topology the Robinson-Wilczek method still works. The horizon is a hypersurface with uniform physical properties, for example the surface gravity and electric potential are constant on the horizon. Our study of black rings shows that quantum field theory near the horizon is relatively simple. In the near horizon limit, $G(y)$ captures the singular structure. After zooming in the horizon, the horizon is effectively a two-dimensional one and the quantum field theory is effectively coupled to the gravitational background (37) after field redefinition. From the analysis of charged black ring we see that near the the horizon the electrodynamics also simplifies, depending only on the radial direction, while outside the horizon it's much more complicated.

The Robinson-Wilczek method has been tested in many cases, at present it does not deal with other aspects of black hole physics such as thermodynamics and entropy, a better understanding on these issues is expected. At least for studying Hawking radiation with Robinson-Wilczek method, we should expect for more general cases the near horizon physics could be simplified and reduced to $(1+1)$ dimensional one, if the horizon is only controlled by a function depending on one coordinate. Though the exploration is still case by case, it seems that this method is quite general.

\section{Acknowledgments}

The work was partially supported by NSFC Grant No. 10405028,10535060, NKBRPC (No. 2006CB805905) and the Key Grant Project of Chinese Ministry of Education (NO. 305001).

Note added:After submit this note we are informed an independent work by Umpei Miyamoto and Keiju Murata [14] which has some overlap with our work.

\section{References}

[1] S.W.Hawking, "Particle Creation By Black Holes," Commun. Math. Phys.43(1975) 199[Erratum-ibid.46(1976)206].

[2] Sean P. Robinson, Frank Wilczek, "Relationship between Hawking Radiation and Gravitational Anomalies," Phys. Rev. Lett. 95 (2005) 011303.arXiv:gr-qc/0502074

[3] Satoshi Iso, Hiroshi Umetsu, Frank Wilczek, "Hawking Radiation from Charged Black Holes via Gauge and Gravitational Anomalies," Phys. Rev. Lett. 96 (2006) 151302. arXiv:hep-th/0602146 
[4] Satoshi Iso, Hiroshi Umetsu, Frank Wilczek, "Anomalies, Hawking Radiations and Regularity in Rotating Black Holes," Phys. Rev. D74 (2006) 044017. arXiv:hep-th/0606018 Keiju Murata, Jiro Soda, "Hawking Radiation from Rotating Black Holes and Gravitational Anomalies," Phys. Rev. D74 (2006) 044018. arXiv:hep-th/0606069

[5] Satoshi Iso, Takeshi Morita, Hiroshi Umetsu, "Quantum Anomalies at Horizon and Hawking Radiations in Myers-Perry Black Holes," JHEP 0704:068,2007. arXiv:hep-th/0612286

[6] Elias C. Vagenas, Saurya Das, "Gravitational Anomalies, Hawking Radiation, and Spherically Symmetric Black Holes," JHEP 0610 (2006) 025.arXiv:hep-th/0606077 M.R. Setare, "Gauge and gravitational anomalies and Hawking radiation of rotating BTZ black holes," Eur. Phys. J. C49:865-868,2007.arXiv:hep-th/0608080 Zhibo Xu, Bin Chen, "Hawking Radiation from General Kerr-(anti)de Sitter Black Holes," Phys. Rev. D75 (2007) 024041. arXiv:hep-th/0612261 Qing-Quan Jiang, Shuang-Qing Wu, "Hawking radiation from rotating black holes in anti-de Sitter spaces via gauge and gravitational anomalies," Phys. Lett. B647 (2007) 200-206.arXiv:hep-th/0701002 QingQuan Jiang, Shuang-Qing Wu, Xu Cai, "Hawking radiation from dilatonic black holes via anomalies," Phys. Rev. D75 (2007) 064029.arXiv:hep-th/0701235 Kui Xiao, Wenbiao Liu, Hongbao Zhang, "Anomalies of the Achucarro-Ortiz black hole," Phys. Lett. B647:482-485,(2007). arXiv:hep-th/0702199 Hyeonjoon Shin, Wontae Kim," Hawking Radiation from Non-Extremal D1-D5 Black Hole via Anomalies," arXiv:0705.0265] Saurya Das, Sean P. Robinson, Elias C. Vagenas, "Gravitational anomalies: a recipe for Hawking radiation", arXiv:0705.2233

[7] Roberto Emparan, Harvey S. Reall, "A rotating black ring in five dimensions," Phys. Rev. Lett. 88 (2002) 101101. arXiv:hep-th/0110260

[8] Henriette Elvang, "A Charged Rotating Black Ring," Phys. Rev. D68 (2003) 124016. arXiv:hep-th/0305247

[9] Henriette Elvang, Roberto Emparan, "Black Rings, Supertubes, and a Stringy Resolution of Black Hole Non-Uniqueness," JHEP 0311 (2003) 035. arXiv:hep-th/0310008

[10] Roberto Emparan, "Rotating Circular Strings, and Infinite Non-Uniqueness of Black Rings," JHEP 0403 (2004) 064. arXiv:hep-th/0402149

[11] Keith Copsey, Gary T. Horowitz, "The Role of Dipole Charges in Black Hole Thermodynamics," Phys. Rev. D73 (2006) 024015.arXiv:hep-th/0505278 D. Astefanesei 
and E. Radu, "Quasilocal formalism and black ring thermodynamics", Phys. Rev. D73 (2006)044014. arXiv:hep-th/0509144

[12] Roberto Emparan, Harvey S. Reall, "Black Rings," Class. Quant. Grav. 23 (2006) R169. arXiv:hep-th/0608012

[13] Maulik K. Parikh, Frank Wilczek, "Hawking Radiation as Tunneling," Phys. Rev. Lett. 85 (2000) 5042-5045.|arXiv:hep-th/9907001

[14] Umpei Miyamoto, Keiju Murata, "On Hawking radiation from black rings," arXiv:0705.3150 